\title{
INÍCIO DA MARCHA NA MIELOMENINGOCELE: UMA REVISÃO INTEGRATIVA
}

\author{
ONSET OF MYELOMENINGOCELE GAIT: AN INTEGRATIVE REVIEW
}

\author{
Francine Zillmer Machado ${ }^{\mathrm{a}^{*}}$, Laís Rodrigues Gerzson ${ }^{\mathrm{b}^{*}}$, Carla Skilhan de Almeida ${ }^{\mathrm{c}^{*}}$ \\ francinefisioterapia@yahoo.com.br ${ }^{\mathrm{a}}$, gerzson.lais@yahoo.com.br ${ }^{\mathrm{b}}$, carlaskilhan@gmail.com \\ Universidade Federal do Rio Grande do Sul (UFRGS)
}

Data de recebimento:13/05/2019

Data de Aceite: 21/10/2019

\section{RESUMO}

Introdução: A mielomeningocele é uma má-formação do sistema nervoso central, onde ocorre a falha do fechamento do tubo neural. Doença congênita, multifatorial, devido de deficiência de ácido fólico. Crianças com este diagnóstico podem apresentar dificuldades urológicas, ortopédicas, neurológicas, entre outras. A marcha pode iniciar em torno de dois anos de idade dificultando o aprendizado motor. Objetivo: Revisar na literatura atual o início da marcha independente e as intercorrências que podem acometer as crianças com mielomeningocele. Materiais e Métodos: Revisão integrativa. Foi realizada busca nas bases de dados PubMed, Lilacs, Scielo, Google Acadêmico, do início das bases até dezembro/2018. A busca compreendeu os descritores "open bífida spine" "myelomeningocele", "gait", "child" associados a seus termos sinônimos e seus equivalentes em inglês. Resultados: Encontrou-se 50 estudos, 35 foram selecionados para análise detalhada, após, oito foram incluídos na revisão. Os dados levantados pela grande maioria das pesquisas concluíram que o segmento mais afetado é a coluna lombar baixa. As intercorrências mais comuns foram úlceras de pressão, luxação de quadril, infecção urinária, fraturas e deformidades Conclusões: Todos os estudos relatam um início atrasado da marcha independente das crianças com MMC em relação às crianças típicas. O início vai depender do nível medular da lesão. A média encontrada foi três anos para lesão lombar baixa e quatro para a torácica. As alterações mais encontradas foram: escoliose, adução de quadril, joelhos fletidos, pés equinos e contraturas que estão relacionadas a assimetria da marcha, fraturas de fêmur e tíbia, luxações de quadril e deformidades nos pés.

Palavras-chave: marcha, mielomeningocele, crianças.

\section{ABSTRACT}

Introduction: Myelomeningocele is a malformation of the central nervous system, where neural tube closure fails. Congenital, multifactorial disease due to folic acid deficiency. Children with this diagnosis may present urological, orthopedic, neurological, and other difficulties. The gait can start around two years old hindering the motor learning. Aim: To review in the current literature the onset of independent gait and the intercurrences that can affect children with myelomeningocele. Materials and Methods: integrative review. We searched PubMed, Lilacs, Scielo, and Academic Google databases from the beginning of the databases until December / 2018. The search comprised the words "open bifida spine", "myelomeningocele", "gait", "child" associated with its synonyms and their English equivalents. Results: 50 studies we found, 35 were selected for detailed analysis, and eight were included in the review. The data collected by the vast majority of studies concluded that the segment most affected is the low lumbar spine. The most common complications were pressure ulcers, hip dislocation, urinary tract infection, fractures and deformities. Conclusions: All studies report a delayed onset of independent gait in children with MMC in relation to typical children. The onset will depend on the medullary level of the lesion. The mean was three years for low back injury and four for thoracic injury. The most frequent alterations were: scoliosis, hip adduction, flexed knees, equine feet and contractures related to gait asymmetry, femoral and tibial fractures, hip dislocations and foot deformities.

Keywords: gait, myelomeningocele, child. 


\section{Introdução}

A mielomeningocele (MMC) ou espinha bífida (EF) aberta é uma malformação embrionária do sistema nervoso central (SNC), que ocorre nas primeiras quatro semanas de gestação. É decorrente de uma falha do fechamento do tubo neural embrionário, ocasionando a exposição da medula espinhal e meninges na superfície dorsal do bebê ao nascimento, caracterizando-se por uma protrusão cística de tecido nervoso exposto. ${ }^{1}$

A MMC é a doença congênita mais comum do SNC compatível com a vida, com incidência global que varia de 0,1 a 10 casos para cada 1000 nascidos vivos ${ }^{2}$ com predominância em recémnatos do sexo feminino e de raça branca, ${ }^{3}$ e entre 0,5 e 1,0 em cada 10.000 nos Estados Unidos. De acordo com os últimos dados publicados pela Organização Mundial da Saúde (OMS), o Brasil é o quarto país com maior taxa de incidência 1,139 a cada 1000 nascidos vivos. ${ }^{4}$ Estudo realizado em Minas Gerais demonstrou prevalência de 4,3 por mil nascimentos, entre $1990-2000^{5}$, e outro, em Recife, constatou prevalência de 5 por mil nascimentos, no período de 2000-2004. ${ }^{6}$

A causa da MMC é multifatorial, porém a deficiência de ácido fólico (AF) é o principal fator de risco. Hipertermia materna durante os estágios iniciais da gravidez, diabetes mellitus, uso de drogas antiepilépticas (ácido valpróico), obesidade e anormalidades cromossômicas podem estar relacionadas com este quadro clínico. Aproximadamente 14\% das crianças nascidas com este diagnóstico morrem antes dos cinco anos de idade e $35 \%$ de mortalidade de crianças com disfunção do tronco encefálico. ${ }^{7}$

É considerada a segunda causa de deficiência do aparelho locomotor em crianças. O grau de incapacidade e gravidade está relacionado com o local em que ocorreu a lesão medular, como também de outros fatores neurológicos, especialmente a hidrocefalia (aumento do liquido cefalorraquidiano nas cavidades cerebrais). A região mais afetada (cerca de $75 \%$ ) é a lombar, mas pode afetar as demais regiões da medula. ${ }^{8}$ O diagnóstico pode ser realizado ainda no período gestacional, o que já favorece um plano de intervenção precoce. Logo após o nascimento, é indicada a cirurgia, com finalidade de reduzir o agravamento do quadro clínico e possíveis sequelas. A cesárea nestes casos é indicada, assim diminuiu as chances de rotura da MMC e de infecção. Exames como a radiografia, ressonância magnética e a tomografia são exames fundamentais para identificar as malformações anatômicas. ${ }^{9}$

Crianças com este diagnóstico podem apresentar dificuldades urológicas, ortopédicas, neurológicas, gastrointestinais, psicossociais e intelectuais. ${ }^{10,11}$ Estes indivíduos apresentam limitações funcionais como: paralisia dos membros inferiores, diminuição da força muscular, atrofia muscular, perda de sensibilidade, tudo isso torna a criança parcial ou totalmente dependente de cuidados. O prognóstico depende do nível medular atingido. Todos esses fatores tornam possíveis ou não, a ortostáse e a deambulação ${ }^{12}$.

Geralmente, o início da marcha ocorre aproximadamente no quarto trimestre de vida da criança típica ${ }^{13}$, sendo que nas crianças com sequelas de MMC a marcha pode iniciar, aproximadamente nos seus dois anos de idade. Sendo assim, a marcha pode ser afetada por diversos motivos, o maior deles é o seguimento neurológico acometido, em decorrência, algumas deformidades ósseas podem surgir, dificultando o processo de aprendizado da deambulação. Quando a lesão ocorre na região torácica, há uma maior propensão de ocorrer escolioses ${ }^{14,15}$ e cifoses. Na região lombar alta é muito comum a luxação de quadril, na região lombar baixa e sacral, deformidades nos pés e ulceras de pressão. Contraturas no quadril e joelhos acometem todos os níveis. Movimentar-se é indispensável para a independência do ser humano. A criança aprende o movimento vivenciando a sensação do mesmo, assim construindo padrões sensório-motores necessários para realizar atividades de vida diária (AVD's). ${ }^{16,17}$

Portanto, o objetivo deste estudo foi revisar na literatura atual o início da marcha independente $\mathrm{e}$ as intercorrências que podem acometer as crianças com mielomeningocele. É muito importante que o profissional que irá acompanhar esta criança entenda exatamente o que pode estar acontecendo. Este estudo traz uma ideia de todo esse processo. 


\section{Materiais e métodos}

Como critérios de elegibilidade, foram incluídos estudossobreoiníciododesenvolvimento da marcha em crianças com meningomielocele e, como desfecho, o início da marcha independente e as intercorrências que podem acometer as crianças com mielomeningocele. Na seleção dos estudos, os títulos e resumos de todos os artigos identificados pela estratégia de busca foram avaliados. Todos os resumos que não forneciam informações suficientes sobre os critérios de inclusão e exclusão foram selecionados para avaliação do texto completo. Nessa segunda fase, foram avaliados os textos completos dos artigos e a seleção foi feita de acordo com os critérios de elegibilidade especificados anteriormente.

Figura 1 - Fluxograma demonstrando a busca e seleção dos estudos

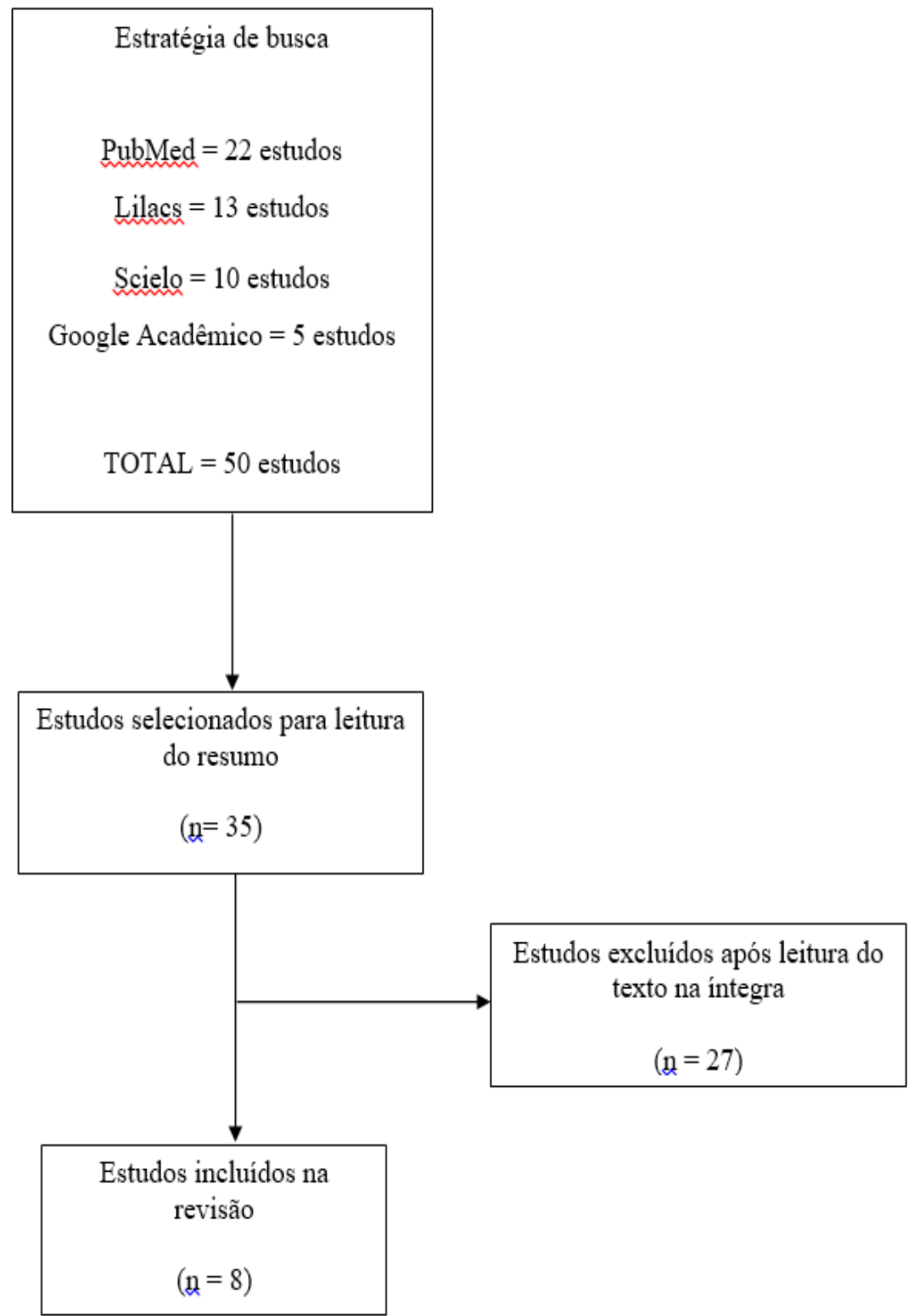

Fonte: autores. 
Como estratégia (Figura 1), foi realizada busca nas bases de dados PubMed, Lilacs, Scielo e Google Acadêmico, de janeiro de 2008 até dezembro 2018, além de busca manual em referências de estudos já publicados sobre o assunto. Selecionaram-se materiais do início das bases até o que a literatura atual apresenta para análise, para não restringir a pesquisa. Os artigos foram selecionados utilizando os seguintes termos: "open bífida spine" "myelomeningocele", "gait", "child" e seus equivalentes em português "espinha bífida aberta", "mielomeningocele", "marcha", "criança", associados a seus termos sinônimos. Os critérios de inclusão do estudo foram: (1) artigos apresentados com texto na íntegra; (2) escritos em português ou inglês; (3) publicados no início das bases até setembro de 2018; (4) estudos com crianças até a segunda infância. Não houve restrições quanto à amostra para maximizar os resultados da pesquisa. Foram excluídos do estudo: (1) monografias (2) artigos de revisão; (3) anais de eventos; (4) dissertações e teses; (5) estudos fora do período do estudo; (6) estudo com animais. A extração dos dados foi realizada utilizando um formulário padronizado. Foram extraídas informações com relação às características metodológicas dos estudos, amostra, idade, características, método de avaliação e desfecho, demonstrados na Tabela 1 .

Tabela 1 - Informações com relação às características metodológicas dos estudos, pacientes, idade, características, método de avaliação e desfecho.

\begin{tabular}{|c|c|c|c|c|c|}
\hline Estudo & Pacientes & Idade & Características & $\begin{array}{l}\text { Métodos de } \\
\text { avaliação }\end{array}$ & Desfecho \\
\hline $\begin{array}{c}\text { Collange } e t \\
a l .,{ }^{18}\end{array}$ & 40 & $\begin{array}{l}3-7,5 \\
\text { anos }\end{array}$ & $\begin{array}{c}\text { Foi avaliada presença } \\
\text { ou ausência de má- } \\
\text { formação Arnold- } \\
\text { Chiari, hidrocefalia, } \\
\text { hidromielia, } \\
\text { medula ancorada e } \\
\text { incontinência vesical } \\
\text { e/ou intestinal. }\end{array}$ & PEDI & $\begin{array}{c}\text { Os níveis de } \\
\text { lesão e aquisição } \\
\text { da deambulação } \\
\text { influenciam de } \\
\text { forma diferente o } \\
\text { desempenho da } \\
\text { criança nas AVD's. }\end{array}$ \\
\hline $\begin{array}{c}\text { Brandão et } \\
a l .,{ }^{19}\end{array}$ & 42 & $\begin{array}{c}8 \mathrm{~m} \\
-4,3 \\
\text { anos }\end{array}$ & $\begin{array}{c}\text { DI, AC, } \\
\text { socioeconômicos, } \\
\text { terapêuticos, } \\
\text { funcionais e } \\
\text { educacionais. }\end{array}$ & $\begin{array}{l}\text { Através dos } \\
\text { prontuários }\end{array}$ & $\begin{array}{l}\text { Ocorrência de LQ } \\
\text { e de fratura possui } \\
\text { associação com os } \\
\text { segmentos afetados } \\
\text { lombar alto e } \\
\text { torácico. }\end{array}$ \\
\hline
\end{tabular}




\begin{tabular}{|c|c|c|c|c|c|}
\hline Estudo & Pacientes & Idade & Características & $\begin{array}{l}\text { Métodos de } \\
\text { avaliação }\end{array}$ & Desfecho \\
\hline $\begin{array}{c}\text { Teulier et } \\
{\text { al. } .{ }^{20}}^{2}\end{array}$ & 24 & $\begin{array}{c}1 \mathrm{~m}, \\
3 \mathrm{~m}, 6 \\
\mathrm{~m}, 9 \mathrm{~m} \\
\text { e } 12 \mathrm{~m} .\end{array}$ & $\begin{array}{l}\text { Foram colocados em } \\
\text { uma esteira no } 1^{\circ} \text { ano } \\
\text { após o nascimento } \\
\text { para comparar a } \\
\text { quantidade de passos } \\
\text { e a qualidade do } \\
\text { pisar. }\end{array}$ & $\begin{array}{c}\text { TP em } 3 \\
\text { condições em } \\
\text { cada criança: RN } \\
\text { pisada, trendmill } \\
\text { stepping e supino. } \\
\text { Sendo colocados } \\
\text { marcadores } \\
\text { fletivos na CI, } \\
\text { trocânter maior, } \\
\text { joelho, ML e RM. }\end{array}$ & $\begin{array}{c}\text { A prática em } \\
\text { esteira rolante } \\
\text { aumentou } \\
\text { a atividade } \\
\text { das pernas, } \\
\text { especificamente na } \\
\text { pisada de bebês, } \\
\text { com capacidade de } \\
\text { respostas variadas } \\
\text { entre lactentes. }\end{array}$ \\
\hline Bartonek $^{21}$ & 43 & $\begin{array}{c}6 m-6 \\
\text { anos }\end{array}$ & $\begin{array}{c}\text { Avaliação de } \\
\text { acompanhamento } \\
\text { no momento do } \\
\text { tratamento. A } \\
\text { abordagem ocorreu } \\
\text { aos } 6 \text { meses, } 1,1.5, \\
\text { 2, } 3,4 \text { e } 6 \text { anos. }\end{array}$ & $\begin{array}{l}\text { FM avaliada } \\
\text { através do teste } \\
\text { em escala com } \\
\text { as diretrizes de } \\
\text { Hislop 23, TT } \\
\text { para amplitude. }\end{array}$ & $\begin{array}{l}\text { Grande variação } \\
\text { no DM antes da } \\
\text { pré-escola entre } \\
\text { crianças com } \\
\text { função muscular } \\
\text { semelhantes. }\end{array}$ \\
\hline Janet et $\mathbf{a l}^{22}$ & 7 & $\begin{array}{l}5-11 \\
\text { anos }\end{array}$ & $\begin{array}{c}\text { Foi utilizado } \\
\text { um estimulador } \\
\text { (Neurotech NT } \\
\text { 200-TES) } 6 \text { noites } \\
\text { por semana. } \\
\text { Eletrodos foram } \\
\text { posicionados em } \\
\text { locais determinados } \\
\text { e o terapeuta instruiu } \\
\text { as famílias através } \\
\text { de telefonemas em } 2 \\
\text { semanas e após em } \\
\text { intervalos mensais } \\
\text { durante o tratamento. }\end{array}$ & $\begin{array}{l}\text { Foram realizados } \\
\text { exames físicos } \\
\text { (teste muscular } \\
\text { manual não } \\
\text { instrumentado do } \\
\text { quadril, flexores, } \\
\text { extensores, } \\
\text { abdutores, } \\
\text { adutores e } \\
\text { joelho flexores } \\
\text { e extensores, } \\
\text { ROM passiva do } \\
\text { quadril, joelhos } \\
\text { e tornozelos e } \\
\text { teste sensorial de } \\
\text { monofilamento } \\
\text { das pernas. }\end{array}$ & $\begin{array}{l}\text { Após os } 9 \text { meses } \\
\text { de tratamento } \\
\text { demonstraram } \\
\text { pequenos ganhos } \\
\text { em FM, marcha } \\
\text { e CI. Mas o uso } \\
\text { do TES não } \\
\text { produziu mudanças } \\
\text { substânciais na } \\
\text { função física } \\
\text { medidas pelo } \\
\text { WeeFIM. }\end{array}$ \\
\hline
\end{tabular}




\begin{tabular}{|c|c|c|c|c|c|}
\hline Estudo & Pacientes & Idade & Características & $\begin{array}{l}\text { Métodos de } \\
\text { avaliação }\end{array}$ & Desfecho \\
\hline $\begin{array}{c}\text { Fujisawa et } \\
{\text { al. },{ }^{23}}^{23}\end{array}$ & 48 & $\begin{array}{l}2-11 \\
\text { anos }\end{array}$ & $\begin{array}{c}\text { Identificar a fase } \\
\text { de DM, a condição } \\
\text { motora, segmento } \\
\text { neurológico, } \\
\text { presença e tipo de } \\
\text { deformidade, uso de } \\
\text { aparelho ortopédico e } \\
\text { assistivos e modo de } \\
\text { locomoção. }\end{array}$ & $\begin{array}{l}\text { Identificação, } \\
\text { socioeconômico, } \\
\text { aspectos } \\
\text { terapêuticos, AF. }\end{array}$ & $\begin{array}{l}\text { O local da lesão } \\
\text { foi determinante } \\
\text { para o prognóstico } \\
\text { da deambulação } \\
\text { em crianças com } \\
\text { mielomeningocele. } \\
\text { Mas não concluí- } \\
\text { ram que os fatores } \\
\text { intervenientes } \\
\text { foram preditores a } \\
\text { capacidade de de- } \\
\text { ambular. }\end{array}$ \\
\hline $\begin{array}{c}\text { Santos et } \\
\text { al. }^{24}\end{array}$ & 51 & $\begin{array}{c}5-16,11 \\
\text { anos }\end{array}$ & $\begin{array}{l}\text { G1: crianças com } \\
\text { idade de } 5 \text { a } 10,11 \\
\text { anos. } \\
\text { G2: crianças com } 11 \\
\text { aos } 16,11 \text { anos. } \\
\text { A divisão de grupos } \\
\text { foi com base } \\
\text { nas idades para } \\
\text { delimitar infância e } \\
\text { adolescência. }\end{array}$ & $\begin{array}{l}\text { Dados pessoais, } \\
\text { intercorrências } \\
\text { clínicas, cirurgias } \\
\text { realizadas, tipo } \\
\text { de marcha, uso } \\
\text { de órteses, grau } \\
\text { de importância } \\
\text { e tempo médio } \\
\text { para aquisição } \\
\text { da marcha. Após } \\
\text { realizado exame } \\
\text { físico: avaliou } \\
\text { integridade da } \\
\text { pele, função } \\
\text { de MsSs, } \\
\text { deformidades em } \\
\text { MsIs e FM dos } \\
\text { FQ e AQ, EJ e FJ. }\end{array}$ & $\begin{array}{c}\text { Os fatores que } \\
\text { influenciam na } \\
\text { duração da marcha } \\
\text { que apresentaram } \\
\text { relevância foram } \\
\text { TM, idade de } \\
\text { início do treino, } \\
\text { quantidade de } \\
\text { cirurgias, presença } \\
\text { de deformidades, } \\
\text { sobrepeso e } \\
\text { motivação. Quanto } \\
\text { mais cedo inicia-se } \\
\text { o TM e mais tempo } \\
\text { permanece em } \\
\text { terapia, maior será } \\
\text { a duração desta } \\
\text { função. }\end{array}$ \\
\hline
\end{tabular}




\begin{tabular}{|c|c|c|c|c|c|}
\hline Estudo & Pacientes & Idade & Características & $\begin{array}{l}\text { Métodos de } \\
\text { avaliação }\end{array}$ & Desfecho \\
\hline $\begin{array}{l}\text { Arazpour } \\
\text { et al..25 }\end{array}$ & 5 & $\begin{array}{l}7-12 \\
\text { anos }\end{array}$ & $\begin{array}{c}\text { Cada criança foi } \\
\text { equipada com uma } \\
\text { IRGO. Receberam } \\
2 \text { h de TM durante } 5 \\
\text { dias por } 8 \text { semanas. } \\
\text { Também foi realizado } \\
\text { alongamento passivo } \\
\text { das extremidades } \\
\text { inferiores, } \\
\text { fortalecimento dos } \\
\text { MsSs e TE com } \\
\text { a órtese em pé e } \\
\text { andando. A marcha } \\
\text { foi analisada } 2 \mathrm{x} \\
\text { no início e após } 8 \\
\text { semanas de TM. }\end{array}$ & $\begin{array}{l}\text { As informações } \\
\text { da marcha foram } \\
\text { obtidas através do } \\
\text { Vicon sistema de } \\
\text { captura, usando } \\
6 \text { câmeras e } 2 \\
\text { plataformas de } \\
\text { força. Marcadores } \\
\text { foram colocados } \\
\text { na órtese. }\end{array}$ & $\begin{array}{l}\text { Encontrou-se } \\
\text { melhora no } \\
\text { treino de marcha } \\
\text { com o IRGO, os } \\
\text { parâmetros de } \\
\text { caminhada em } \\
\text { comparação com } \\
\text { a linha base nos } \\
\text { assuntos da MMC. }\end{array}$ \\
\hline
\end{tabular}

PEDI: Avaliação Pediátrica do inventário de incapacidade; AVD's: atividades de vida diária, DI: dados de identificação; AC: aspectos clínicos; LQ: luxação de quadril; TP: testes de pisada; RN: recém-nascido; CI: crista ilíaca, ML: maléolo lateral, RM: região metatársica; FM: força muscular; TT: testes de Thomas; DM: desenvolvimento motor; TES: limiar de estimulação elétrica; ROM: range of motion; CI: continência intestinal; WEEFIM: Medida de Independência Funcional para Crianças; AF: avaliação fisioterapêutica; G: grupo; MsSs: membros superiores, MsIs: membros inferiores; FQ: flexores de quadril; AQ: adutores de quadril; EJ: extensores de joelho; FJ: flexores de joelho; TM: treino da marcha; IRGO: órtese de marcha recíproca isocêntrica; TE: treino de equilíbrio; MMC: Mielomeningocele.

Fonte: autores

\section{Resultados}

\section{Descrição dos estudos}

A estratégia de busca identificou 50 artigos, dos quais 35 foram considerados relevantes e retomados para análise detalhada; destes, 27 foram excluídos: 16 por se tratarem de artigos de revisão bibliográfica, nove por estarem fora da data estabelecida e dois por serem anais de eventos. Oito estudos (três do Lilacs, um do Scielo, um do Google acadêmico e três do PubMed) preencheram os critérios de elegibilidade e foram incluídos nesta revisão. Destes estudos utilizados, dois eram estudos retrospectivos, quatro eram estudos transversais prospectivos e um estudo piloto. Em um dos estudos apresentou dificuldade em manter a participação de todas as crianças que compunham a amostra até o final. ${ }^{22}$

Os dados levantados pela grande maioria das pesquisas concluíram que o segmento mais afetado é a coluna lombar baixa. ${ }^{23}$ Já a musculatura mais afetada pela fraqueza foi quadríceps femoral, músculos tibiais e gastrocnêmio. As intercorrências mais comuns foram úlcera de pressão, infecção urinária, luxação de quadril, fraturas e deformidades. ${ }^{19,23}$ Houve associação entre lesão torácica e fraturas, já em pacientes com lesão lombar alta a associação foi entre a luxação de quadril. ${ }^{19}$ Em relação ao gênero, a predominância foi de meninas na maioria dos estudos. ${ }^{18,19,23,24}$

Levasse em conta aárea neurológica afetada para determinar possíveis deambuladores. Crianças com lesão torácica e lombar alta necessitaram de cadeira de rodas, aquelas com lesão lombar baixa as crianças eram deambulantes. ${ }^{23}$ Uma comparação entre o início da marcha entre crianças típicas e crianças com lesão lombar baixa constatou-se que as crianças com está lesão iniciaram a marcha aos três anos e dez meses e a criança típica no seu primeiro ano de vida. ${ }^{21} \mathrm{O}$ estudo que investigou a utilização de órtese longa (OL) para o treino de 
marcha, observou que o tempo para adquirir a marcha é de dois anos e dois meses, sendo contado a partir do início do treino de marcha. ${ }^{24}$

Com relação aos métodos avaliativos utilizados pelos profissionais, cada estudo realizou sua pesquisa de forma distinta, mas buscando o objetivo de averiguar o tempo de marcha para cada lesão e os fatores de maior interferência para o aprimoramento da marcha. ${ }^{18,19,20,21,22,23,24,24}$. A Tabela 2 mostra o que os estudos selecionados relatam sobre o início da marcha.

Tabela 2 - Início da Marcha.

\begin{tabular}{|c|c|}
\hline Estudo & Início da Marcha \\
\hline Collange et al., ${ }^{18}$ & $\begin{array}{l}\text { Foram selecionadas crianças a partir dos três anos de idade, pois nessa } \\
\text { idade já apresentam nível de deambulação. }\end{array}$ \\
\hline Brandão et al., ${ }^{19}$ & $\begin{array}{l}\text { A deambulação acontece em diferentes idades, de acordo com o segmento } \\
\text { afetado. Lesão torácica com quatro anos e seis meses, lombar-alto aos } \\
\text { cinco anos e dois meses, lombar-baixa aos três anos e } 10 \text { meses e sacral } \\
\text { aos dois anos e } 2 \text { meses }\end{array}$ \\
\hline Teulier et al., ${ }^{20}$ & $\begin{array}{l}\text { Neste estudo a capacidade de andar aconteceu aos } 24 \text { meses, } 29 \text { meses e } \\
44 \text { meses. }\end{array}$ \\
\hline Bartonek $^{21}$ & $\begin{array}{l}\text { Crianças com lesão em nível lombar baixo a marcha foi alcançada com } \\
\text { idade média de três anos } 10 \text { meses. }\end{array}$ \\
\hline Janet et $\mathrm{al}^{22}$ & Este estudo não cita idades para o início da marcha \\
\hline Fujisawa et al., ${ }^{23}$ & $\begin{array}{l}\text { Crianças com mielomeningocele adquiriram a capacidade de andar em } \\
\text { torno de } 37 \text { meses de idade. }\end{array}$ \\
\hline Santos et al. ${ }^{24}$ & $\begin{array}{l}\text { O início do treino de marcha teve idade média de quatro anos e cinco } \\
\text { meses e idade média de início da marcha foi aos seis anos e seis meses. }\end{array}$ \\
\hline Arazpour et al., ${ }^{25}$ & Este estudo não cita idades para o início da marcha. \\
\hline
\end{tabular}

Fonte: autores

\section{Discussão}

A MMC é uma má-formação do sistema nervoso central (SNC), onde não ocorre o fechamento correto do tubo neural. Crianças afetadas por essa má-formação demonstram desenvolvimento mais lento, onde cerca de 69\% das crianças necessitam de assistência para suas atividades. ${ }^{18} \mathrm{O}$ presente estudo pretendia revisar na literatura atual o início do desenvolvimento da marcha e as intercorrências que podem acometer as crianças com mielomeningocele. No entanto, os estudos mostram que cada criança irá evoluir de modo individual, independente de sua idade e nível de lesão. Decidir o momento de iniciar o treino de marcha também deve ser de acordo com os resultados que o lactente vem apresentando. ${ }^{21}$

Em relação à marcha independente, crianças com mielomeningocele tiveram o início do deambular atrasado dois anos em comparação com seus pares em desenvolvimento típico. ${ }^{20}$ Também, bebês com mielomeningocele apresentam a média 
de tempo de passadas sem evolução quando comparados aos bebês com desenvolvimento típico, que obtiveram um aumento do número de passos com o passar dos anos. ${ }^{20}$

Fujisawa $^{23}$ ressalta que a criança com mielomeningocele começa a caminhar em torno de 37 meses, ou seja, por volta de três anos de idade. Até então, não é possível definir se a criança dependerá ou não do uso de cadeira de rodas. Em outro estudo, o autor relata que a deambulação ocorre em diferentes idades e depende do segmento afetado, onde a conquista da marcha pode ocorrer em torno dos quatro anos se a lesão for na região torácica; aos cinco anos se a lesão for na região lombar alta; aos três anos e dez meses se a lesão for na região lombar baixa e dois anos na região sacral. A aquisição da marcha em lesão de nível lombar é inversamente proporcional à altura da má-formação ${ }^{19}$.

Santos et al., ${ }^{24}$ constataram em seu estudo que, quanto mais avançada a idade da criança, menor será o tempo para adquirir a função da marcha. Foi encontrada relação entre o segmento afetado e a idade para as formas de locomoção. Crianças com lesão alta eram cadeirantes e crianças com lesão baixa eram deambulantes. ${ }^{19}$ A lesão de nível torácico é considerado o comprometimento mais complexo, pois existe uma paralisia flácida nos quadris, podendo afetar inclusive a musculatura do tronco. ${ }^{18}$ Em relação a cuidados pessoais, pacientes com lesão torácica são os que apresentam os piores resultados funcionais. ${ }^{19}$

Notou-se a importância de determinar logo após o nascimento o nível do envolvimento neurológico, o que tornaria mais fácil estimular o potencial motor e já prevenir as deformidades. ${ }^{20}$ Para o alcance de uma marcha efetiva, conquistar estabilidade na fase de apoio é de suma importância. É muito comum que pacientes com mielomeningocele apresentem um excessivo desvio vertical do centro de gravidade e, se não houver interferência, este fator pode vir a causar assimetria da marcha. Quanto mais função muscular os membros apresentarem, mais cedo se alcançará a posição ortostática ${ }^{21}$.

$\mathrm{Na}$ pré-escola existe uma grande variação entre as crianças em relação ao desenvolvimento da função muscular. Tem se considerado um ato intuitivo determinar a maturação e o momento certo de iniciar o treino de marcha de uma criança considerando que crianças com diferentes níveis de lesão irão progredir de formas diferentes. ${ }^{21}$

O uso de estimulação muscular tectônica, utilizado para fraqueza muscular tem sido considerado um bom recurso, mas por muitas vezes não é tolerado pela criança. Em um estudo, a aplicação do limiar de estimulação elétrica (TES) ocorreu no período da noite, melhorando a força muscular e a função, porém não encontraram mudança significativa no movimento articular e na sensação das extremidades inferiores. $\mathrm{O}$ uso de estimulação elétrica neuromuscular vem sendo utilizado para o tratamento de musculaturas atróficas e fracas ${ }^{22}$.

$\mathrm{O}$ treino em esteira se mostrou eficiente. A prática fez com que houvesse uma maior atividade motora. Este tipo de treino pode oferecer maiores experiências, acelerando o desenvolvimento de estruturas ósseas, colaborando para o controle motor. $^{20}$

$\mathrm{O}$ uso de algum aparato ortótico pode ser necessário. $\mathrm{O}$ uso de órtese de marcha recíproca isocêntrica (IRGO) demonstrou evolução significativa nos movimentos, melhorando as ações compensatórias em relação à linha de base, e também reduziu o gasto energético. ${ }^{25} \mathrm{~A}$ órtese sólida tornozelo-pé (AFO) é normalmente indicada para crianças com MMC, pois colabora para diminuição do grau de dorsiflexão, e favorece a marcha da criança com lesão elevada. Destacase também a importância do uso de muletas canadenses, como auxílio para a marcha. É necessário inclusive que usuários de cadeira de rodas façam uso de algum dispositivo que para o ortostatismo, o que trará benefícios fisiológicos. ${ }^{23}$

Estudo mostrou que o uso de órteses longa (OL) para crianças com MMC também tem sido indicado para o auxílio da marcha. Pacientes com idade média de 6,6 anos e que fazem uso da OL têm adquirido marcha em menor tempo de treino; a justificativa para isso é a maior autonomia que crianças mais velhas possuem ${ }^{24}$. Fatores como deformidades, obesidade, aumento do gasto energético e falta de acessibilidade, faz com que haja um grande número de pacientes que abandonam o uso desta órtese. Procedimentos ortopédicos têm apresentado benefícios em relação ao uso da órtese. 
No entanto, a MMC é uma patologia que provoca muitas contraturas e deformidades. Quando o paciente apresenta contraturas e deformidades, o uso da órtese torna-se difícil, sendo a cirurgia ortopédica uma boa opção para a melhora da função. ${ }^{24}$ Deformidades foram encontradas em maior número em crianças não deambuladoras. Essas deformidades aparecem com mais frequência em quadril e pés. Em crianças mais velhas, as deformidades se acentuam na coluna e joelhos ${ }^{24}$. Para que haja aquisição da marcha, existe a necessidade de motivação principalmente por parte do paciente, onde a busca pela funcionalidade se torna muitas vezes difícil devido ao surgimento das deformidades. ${ }^{24}$

As alterações da articulação do quadril, reduzida velocidade média de caminhada e comprimento da passada na criança com MMC foi verificada quando comparado a criança com desenvolvimento típico após um treinamento de marcha. ${ }^{25}$

Podemos considerar a fraqueza muscular como umas das intercorrências que mais afetam crianças com mielomeningocele. ${ }^{22}$ Se encontram nos pacientes com MMC mais frequentemente deformidades como: escoliose, adução de quadril, joelhos fletidos, pés equinos e contraturas que estão relacionadas a assimetria da marcha. Fraturas em fêmur e tíbia também possui relação com a área de lesão, estas fraturas ocorrem pela baixa densidade mineral, devido à falta de movimento em membros inferiores. ${ }^{23}$

Essas deformidades acontecem de acordo com o segmento afetado. Crianças com lesão torácica são propensas a escolioses e hipercifoses; crianças com lesão lombar alta são propensas às luxações de quadril e, nas lesões lombar baixa e sacral, é mais comum as deformidades nos pés. ${ }^{19}$ Outro estudo que avaliou as habilidades funcionais relata a necessidade de assistência máxima nas atividades de locomoção nos pacientes que não realizam deambulação. Sendo os pacientes com lesão em nível torácico os que apresentam os piores resultados funcionais. ${ }^{18}$

A principal limitação encontrada foi a escassez de pesquisas na infância e primeira infância. A grande maioria dos estudos analisados era com adolescente e artigos de revisão, que não entraram neste estudo. Além disso, a pesquisa analisou os artigos dos últimos 10 anos, o que de certa forma torna-se também uma limitação importante.

É notório que as intercorrências, como fraqueza muscular, fraturas de fêmur e tíbia e escolioses, interferem nas conquistas em relação a marcha, tornando-a muitas vezes assimétrica. Os estudos chegam ao consenso de que a média de idade para o início da marcha é de dois anos e meio após o início do treino de deambulação. Para se obter melhores resultados, quanto mais cedo se inicia a estimulação à marcha maior será a permanência dessa aquisição. É necessário que as famílias tenham o entendimento da necessidade de iniciar a terapia cedo para se ter uma melhor evolução motora, já o profissional responsável pela criança precisa ter bom embasamento para ofertar o melhor tratamento e evitar as deformidades.

Percebe-se a necessidade da realização de pesquisas com crianças ainda no inicio do desenvolvimento motor, para que se obtenha maior conhecimento sobre este período, que se considera muito importante.

\section{Conclusão}

Conhecer as intercorrências que podem acometer o desenvolvimento da marcha na mielomeningocele se mostrou de suma importância, tendo em vista que, oferecer tratamento adequado e precoce, pode excluir ou minimizar os surgimentos dessas intercorrências. Portanto, um diagnóstico precoce torna a intervenção precoce e mais efetiva.

Todos os estudos relatam um início atrasado da marcha independente das crianças com MMC em relação às crianças típicas. Também, esse início vai depender do nível medular da lesão. Quanto mais alto, mais pode demorar a deambular, ou mesmo, a criança pode tornar-se cadeirante. A média encontrada foi três anos para lesão lombar baixa e quatro para a torácica. É muito importante o trabalho de força muscular, dando ênfase aos músculos antigravitários. O treino em esteiras pode antecipar a marcha. $\mathrm{O}$ uso de órteses e muletas canadenses podem auxiliar no processo e não permitir que contraturas e deformidades se instalem. As alterações mais encontradas foram: escoliose, adução de quadril, joelhos fletidos, pés 
equinos e contraturas que estão relacionadas a assimetria da marcha. Fraturas em fêmur e tíbia também foram encontradas. Crianças com lesão torácica são propensas a escolioses e hipercifoses; crianças com lesão lombar alta são propensas às luxações de quadril e, crianças com lesão lombar baixa e sacral, é mais comum às deformidades nos pés.

\section{Referências}

1. Fieggen G, Fieggen K, Stewart C, Padayachy L, Lazarus J, Donald K, et al. Spina bifida: A multidisciplinary perspective on a many-faceted condition. S Afr Med J. 2014;104(3):213-7.

2. Freitas GL, Sena RR, Braga DCO, Faleiros F. Reabilitação de crianças e adolescentes com mielomeningocele: relato de experiência de atuação da enfermagem. Cienc Cuid Saude. 2016;15(4):768-773.

3. Borba LAB, Silva PEC, Junior JOZ, França FV. Perfil clínico-epidemiológico dos pacientes tratados com mielomeningocele em um hospital universitário de Curitiba. Arq Bras Neurocir. 2012; 31(4): 195-9.

4. WHO. World. Atlas of Birth Defects. 2nd ed. Geneva2003. p. 237.

5. Aguiar MJB, Campos AS, Aguiar RALP, Lana AMA, Magalhães RL, Babeto LT. Defeitos de fechamento do tubo neural e fatores associados em recém-nascidos vivos e natimortos. J Pediatr. 2003;79(2):129-34.

6. Pacheco SS, Souza AI, Vidal AS, Guerra GVQL, Filho MB, Baptista EVPB, et al. Prevalência dos defeitos de fechamento do tubo neural em recém-nascidos do Centro de Atenção à Mulher do Instituto Materno Infantil Prof. Fernando Figueira, IMIP: 2000-2004. Rev Bras Saúde Matern Infant. 2006; 6(1):35-42.

7. Salomão RM, Cervante TP, Salomão JFM, Leon SVA The mortality rate after hospital discharge in patients with myelomeningocele decreased after implementation of mandatory flour fortification with folic acid. Arq Neuropsiquiatr. 2017;75(1):20-24

8. Lima CB, Ferreira ES, Bonifacio MR, Silva RC, Santos SS, Alvarenga ML. Avaliação antropométrica em crianças com mielomeningocele. RBNE. 2015; 9(52):336-341.

9. Warf BC. Hydrocephalus associated with neural tube defects: characteristics, management, and outcome in sub-Saharan Africa. Childs Nerv Syst. 2011;27(10):158994.

10. Lamônica DAC, Ferreira AT, Prado LMD, Crenitte PDAP. Desempenho psicolinguístico e escolar de irmãos com mielomeningocele. Rev. CEFAC. 2012; 14(4):763-769.
11. Figueiredo SV, Gomes ILV, Queiroz MVO, Mota DDDS, Sousa ACCD, Vasconcelos CMP. Families' knowledge about children and adolescents with neural malformation about their rights in health. Esc. Anna Nery Rev. Enferm. 2015;19(4):671-678.

12. Freitas GL, Sena RR, Faleiros F, Faleiros JC. Rehabilitation of children and adolescents with myelomeningocele: the daily life of mothers-caregivers. Rev Gaúcha Enferm. 2016;37(4): e60310.

13. Gerzson LR, Azevedo KA, Demarco PR, Catarino BM, Palma MS, Almeida CK.O berçário da escola pública: aplicação de um programa de intervenção motora oportuna. Fisioterapia Brasil 2017;18(4):417 -425.

14. Thomas JGHwang SWBlumberg TJWhitehead WECurry DJLuerssen TG: Correlation between shunt series and scoliosis radiographs in children with myelomeningoceles. J Neurosurg Spine. 2012; 17(5):410-4.

15. Mummareddy N, Dewan MC, Mercier MR, Naftel RP, Wellons JC, Bonfield CM. Scoliosis in myelomeningocele: epidemiology, management, and functional outcome. J Neurosurg Pediatr. 2017; 20(1):99-108.

16. Sandler AD. Children with spina bifida: key clinical issues. Pediatr Clin North Am. 2010;57(4):879-92.

17. Façanha DMA. Avaliação da Funcionalidade em Crianças com Mielomeningocele [Dissertação]. Fortaleza: Universidade Federal do Ceará - UFC; 2015.

18. Collange LA, Franco RC, Esteves RN, Collange NZ. Desempenho funcional de crianças com mielomeningocele. Fisioter. Pesqui. 2008; 5(1): 58-63.

19. Brandão AD, Fujisawa DS, Cardoso JR. Características de Crianças com Mielomeningocele: implicações para a fisioterapia. Fisioter. Mov. 2009;22(1):69-75.

20. Teulier C, Smith BA, Kubo M, Chang CL, Moerchen V, Murazko K et al. Stepping Responses of Infants with Myelomeningocele when Supported on a Motorized Treadmill. Phys Ther.2009; 89(1):60-72.

21. Bartonek A. Motor Development Towrad Ambulation in Preschool Children with Myelomeningocele- A prospective study. Pediatr Phys Ther. 2010;22(1):52-60.

22. Janet L, Walker MD, Shelley W, Ryan PT, Tonya R. Does Threshold Nighttime Electrical Stimulation Benefit Children With Spina Bifida? A Pilot Study. Clin Orthop Relat Res. 2011; 469(5):1297-301.

23. Fujisawa DS, Gois MLCC, Dias JM, Alves EONM, Tavares MS, Cardoso JR. Intervenção de Fatores na Caminhada de Crianças com Mielomeningocele. Fisiot. Movim. 2011;24(2): 275-283

24. Santos FM, Barbosa EC, Pinheiro DL, Torini KA, Chang AL, Justo AB. A Aquisição e Permanência da Marcha com Órtese Longa na Mielomeningocele Nível Lombar Alto. Rev Neurocienc 2013;21(1):28-35. 
25. Arazpour M, Soleimani F, Sajedi F, Vameghi R, Boni MA, Gharib M, Samadian M. Effect of Orthotic Gait Training with Isocentric Reciprocating Gait Orthosis on Walking in Children with Myelomeningocele. Top Spinal Cord Inj Rehabil.2015;23(2):147-154.

\section{Como citar este artigo:}

Machado FZ, Gerzson LR, Almeida CS. Início da marcha na mielomeningocele: uma revisão integrativa. Rev. Aten. Saúde. 2019; 17(61): 93-104. 